\title{
Extremum seeking control techniques applied to photovoltaic systems with multimodal power curves
}

\author{
Waleed Nwesaty; Antoneta Iuliana Bratcu, Member, IEEE; and Ahmad Hably \\ Grenoble Institute of Technology, GIPSA-lab, Control Systems Department, 11 Rue des Mathématiques, Grenoble Campus \\ BP46, F-38402, Saint-Martin d'Hères, France
}

waleed.nwesaty@gipsa-lab.grenoble-inp.fr; antoneta.bratcu@gipsa-lab.grenoble-inp.fr; $\underline{\text { ahmad.hably@gipsa-lab.grenoble-inp.fr }}$

\begin{abstract}
This paper proposes a modified Perturb and Observe (P\&O) Extremum Seeking Control (ESC) technique in presence of multiple maxima. ESC is applied to single-phased gridconnected photovoltaic (PV) arrays which have to provide maximum power irrespective of solar irradiance conditions. In particular, partially shadow conditions may lead to steady-state power curves exhibiting multiple maxima. The power harvested from the PV generator is injected in the single-phased power grid by using two power converter stages: step-up DC-DC converter and DC-AC inverter. When multiple power maxima exist, the amplitude of the perturbation signal plays an important role in successfully tracking the global maximum. Two amplitude modulation strategies are analyzed for the same case study: amplitude modulation by using a first-order-system-response signal and amplitude modulation by using small duty ratio square-wave signal, respectively. MATLAB ${ }^{\circledR} /$ Simulink $^{\circledR}$ numerical simulations are presented in order to assess the two approaches comparatively.
\end{abstract}

Keywords: renewable energy systems, Extremum seeking control; optimal control; cascade control; hill climbing; modulation; perturb and observe.

\section{INTRODUCTION}

$\mathrm{O}$ ne of the most important issues all over the world is how to improve efficiency of renewable energy systems (solar, wind, tidal, etc.). Many researches focus on increasing the efficiency of these energy systems while the energy consumption is also increasing [1], [2]. Among such systems, the photovoltaic (PV) generators nowadays record important growth in terms of harvested power and penetration into the grid [3]. Many cells are grouped in one module (or panel), and many modules form a PV generator. A PV module has nonlinear steady-state characteristics, expressed as either current versus voltage (the so called $I-V$ curve), or as power versus voltage (the $P$ - $V$ curve, like the one in Fig. 1). The $I-V$ and $P-V$ curves of a PV system vary with the solar insulation (irradiance) and cell temperature [4].

In grid applications, it is required to harvest the maximum power from the PV system in order to inject it into the grid [3]. The P-V curve contains a single maximum power point (MPP) when balanced irradiance is applied for all modules of the PV generator, but this is not the case when the irradiance is imbalanced due to a cloud, tree or even the insulation angle, that is, the P-V curve will have multiple peaks expressed as local and global MPP and it is desired to operate the PV module close to the global one. This process is called Maximum Power Point Tracking (MPPT). This MPPT algorithm ideally should ensure working in the global maximum one, but until now it is a challenge to make online identification for the global power point whatever the surrounded conditions.

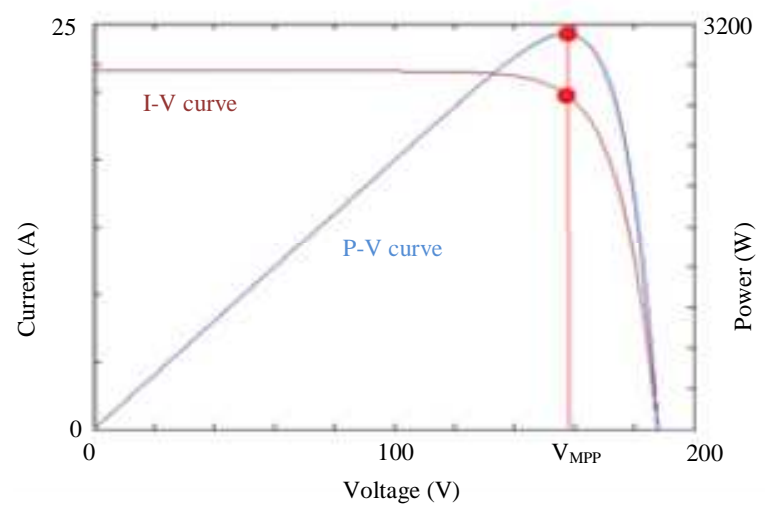

Fig. 1. Photovoltaic cell $I-V$ and $P-V$ characteristics (in red and blue respectively) with the maximum power point.

In the literature plenty of MPPT algorithms are proposed for such systems in order to harvest maximum power: shortcircuit photovoltaic generator method, open-circuit voltage photovoltaic cell test method, incremental conductance method (CI) [5], voltage-offset resistive control (VRC) [6], multivariable gradient-based ES [7], integer-order extremum seeking control (IOESC) [8]. Perturb and observe (P\&O) is one of the most used classes of such algorithms because of its simplicity. It perturbs the operating point of the PV generator and observes the output power; depending on this observation, the operating point is "pushed" in the direction of growing power until the maximum point is reached when $\frac{d P}{d V}=0$. Classical P\&O method uses fixed perturbation amplitude; its performance must be sized as a tradeoff between transient rise 
time and steady-state error [9].

In this paper a modified $\mathrm{P} \& \mathrm{O}$ algorithm is presented and checked by numerical simulation. Unlike the classical $\mathrm{P} \& \mathrm{O}$, this algorithm can ensure working in the desired maximum power point in case of multiple maxima if proper parameters are chosen without having high steady state error. The basic idea is to modulate the perturbation signal's amplitude. Two types of such amplitude modulation are tested in this paper; their advantages and drawbacks when applied to MPPT control are assessed by numerical simulation. Thus, the MPPT algorithm performance and its efficiency are tested under different scenarios of irradiance variation (e.g., corresponding to different types of cloudy weather).

This paper is structured as follows: In section II the structure of the considered PV system is presented and the associated control objectives are formulated. In Section III, the PV source and its associated DC -DC converter are modeled. Section IV details the two-level control strategy and the two ESC algorithms with perturbation signal amplitude modulation. Section V discusses some illustrative simulation results under various daylight scenarios. The paper ends with conclusion and future work.

\section{SYSTEM CONFIGURATION AND CONTROL GOALS}

The studied PV power generator consists of 16 panels, each of which containing $72 \mathrm{PV}$ cells connected in series. 4 panels are connected together in series forming a row, and there are 4 such rows connected in parallel (Fig. 2). This system can produce $3 \mathrm{~kW}$ while it is working in reference conditions (irradiance $1000 \mathrm{~W} / \mathrm{m}^{2}$ and temperature $25{ }^{\circ} \mathrm{C}$ ). The PV generator is connected to a boost (step-up) DC-DC converter, which allows controlling the output voltage of the PV generator. As grid application is considered, the boost converter is followed by an inverter in charge with regulating the DC-link voltage. Finally, the inverter is connected to the power grid. Cascade control strategy is chosen in order to control the system (Fig. 3). The control structure consists of two level control loops: low-level loop contains two sub-loops, the PV voltage control loop and the boost converter current control loop, and high-level loop controls the PV power. In the low-level, the internal variables are controlled in order to operate the system in a desired working point; while in the high level, the desired working point is achieved according to the ESC algorithm.

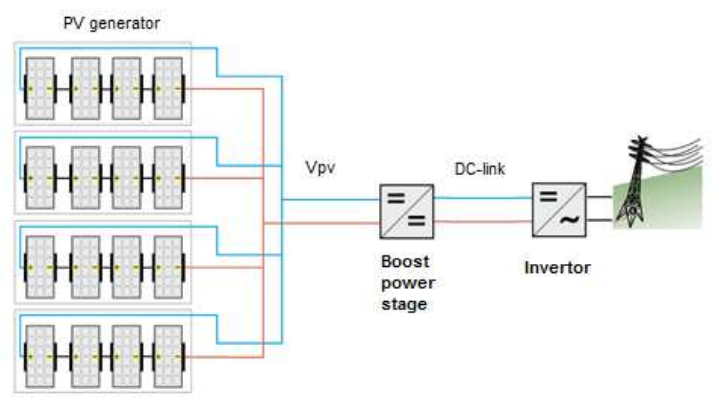

Fig. 2. Grid-connected PV energy conversion system with series- and parallel-connected panels.

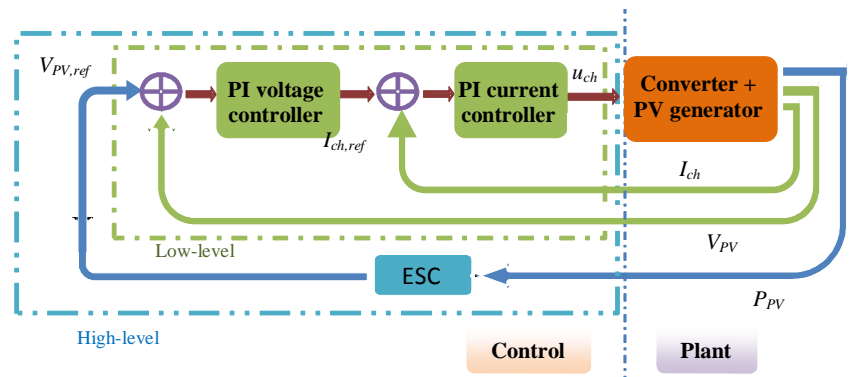

Fig. 3. Cascade control strategy of the PV system.

\section{SYSTEM MODELING}

The system consists of three main parts as described in Fig. 4 [10]: the PV generator, the DC-DC boost converter, the DC-AC angle phased inverter (H-bridge) and the power grid.

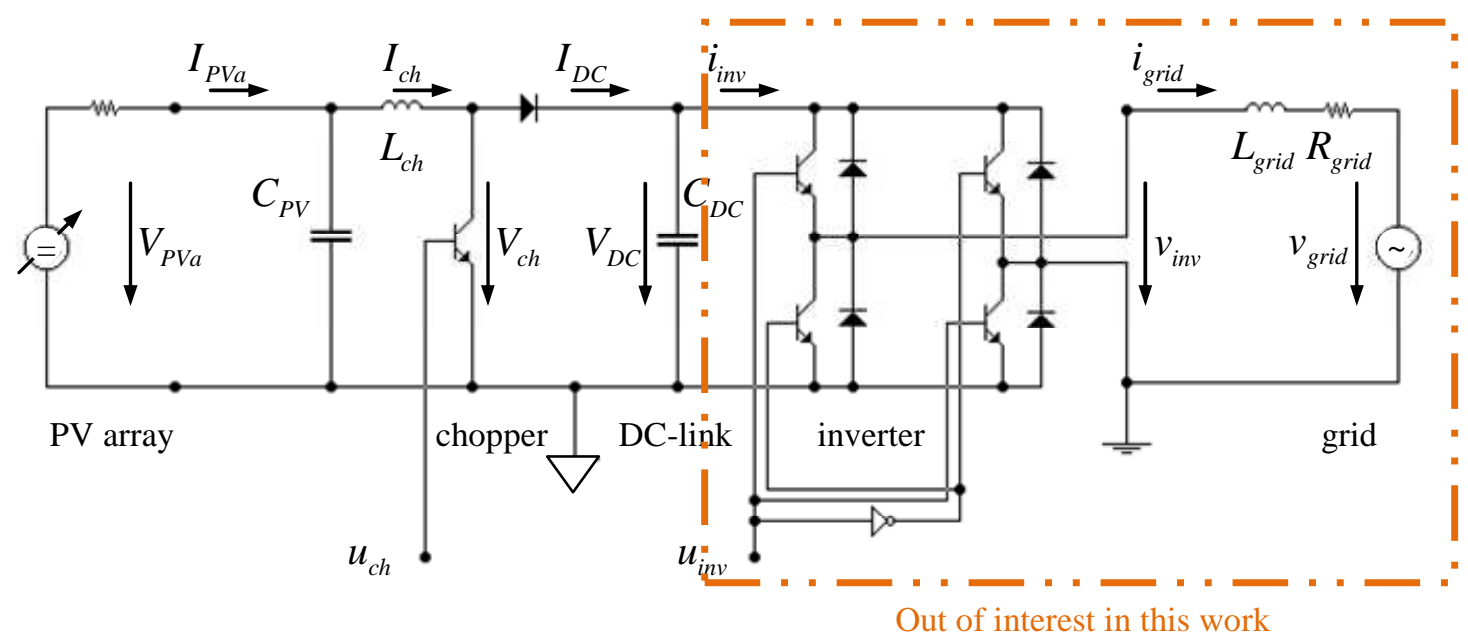

Fig. 4. Electrical scheme of the two-stage PV energy conversion system emphasizing the boost converter electrical scheme [10]. 
In order to investigate the behavior of the ESC algorithm, the first two parts should be studied. It is supposed that the inverter works well and keeps the DC-link voltage constant in order to convey all the available power into the power grid; its operation is not of interest in this paper.

\section{A. PV Generator Modeling}

The basic element in the PV generator is the PV cell, modeled by using the 5-parameter model, which is an equivalent electrical circuit with the same nonlinear behavior of the PV cell (Fig. 5) [2] , [6] , [11].

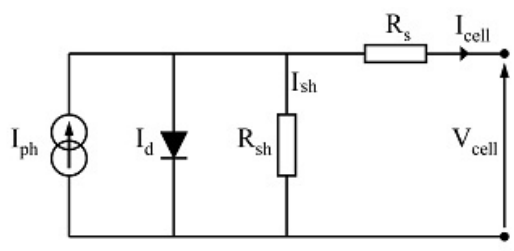

Fig. 5. 5-parameter model of a PV cell.

The 5-parameter model is described by:

$$
\begin{aligned}
& I_{\text {cell }}=I_{p h}-I_{o}\left(e^{\frac{V_{\text {cell }}+R_{s} \cdot I_{\text {cell }}}{V_{t}}}-1\right)-\frac{V_{\text {cell }}+R_{s} \cdot I_{\text {cell }}}{R_{\text {sh }}} \\
& I_{p h}=\frac{G}{G_{r e f}}\left[I_{c c, \text { ref }}+\alpha\left(T_{\text {cell }}-T_{r}\right)\right], \\
& I_{o}=I_{o r}\left(\frac{T_{\text {cell }}}{T_{r}}\right)^{3} e^{\left[\frac{q \cdot \text { Eg }}{k}\left(\frac{1}{T r}-\frac{1}{T_{\text {cell }}}\right)\right]}, \quad V_{t}=\frac{n \cdot k \cdot T_{\text {cell }}}{q},
\end{aligned}
$$

where $G$ and $T_{\text {cell }}$ are the irradiance level and the cell temperature respectively, determined according to the climate conditions, $R_{s}$ and $R_{s h}$ are series and shunt resistances respectively, being related to the type of used PV cell, and $V_{\text {cell }}$ is the cell voltage as imposed by the DC-DC converter. $I_{c c, r e f}$ is the short-circuit current and $I_{o r}$ is the diode inverse saturation current reference, $q$ is the electron charge, $E_{g}$ is the silicon energy gap, $k$ is the Boltzman's constant and $n$ is the diode ideality factor.

\section{B. DC-DC Converter Modeling}

The PV generator is followed by a DC-DC converter which imposes an operating point; it steps up the voltage to fulfill the requirements of the next stage. The boost converter structure is illustrated in Fig. 4.

It is considered that the DC-DC converter works in continuous conducting mode (its current $I_{c h}$ never becomes zero). The converter is controlled in input-voltage-tracking mode (its input voltage i.e., the PV output voltage $V_{P v a}$ to follow variations imposed by the upper control level, the ESC MPPT algorithm). The model is given by:
$V_{P v a}=\left(I_{P v a}-I_{c h}\right) \frac{1}{C_{P v} \cdot s}, \quad I_{c h}=\frac{V_{P v a}-\left(1-u_{c h}\right) V_{D C}}{R_{c h}+L_{c h} \cdot s}$

With $I_{P v a}$ being the PV generator current, $u_{c h}$ being the PWM control signal and $V_{D C}$ being the DC-link constant voltage maintained by the grid-tie inverter (following stage).

\section{CONTROL DESIGN}

The two levels of the control structure in Fig. 3 are designed as it is detailed next.

\section{A. Low-level Control Loops}

Two nested control loops are used, the converter current control loop and the PV generator voltage control loop respectively. The converter current is controlled in order to ensure the continuous-conduction mode of the converter and to avoid over-currents which are harmful for the converter components. To this end, a PI controller is used, ensuring zero steady-state error. The PI controller is tuned to satisfy closed-loop fast response (e.g., 10 times faster than the response of the voltage closed loop) without overshoot.

The outer PI-controller-based closed loop is used to ensure tracking the voltage reference imposed by the ESC algorithm, thus establishing the system's desired working point.(Fig.3) The controller is tuned in order to have fasttime closed-loop response (e.g., 10 times faster than the ESC loop) without overshoot. Both PI controllers are tuned based on linearizing the steady-state $I-V$ curves around the MPP point and under the reference conditions (irradiance 1000 $\mathrm{W} / \mathrm{m}^{2}$ and temperature $25^{\circ} \mathrm{C}$ ).

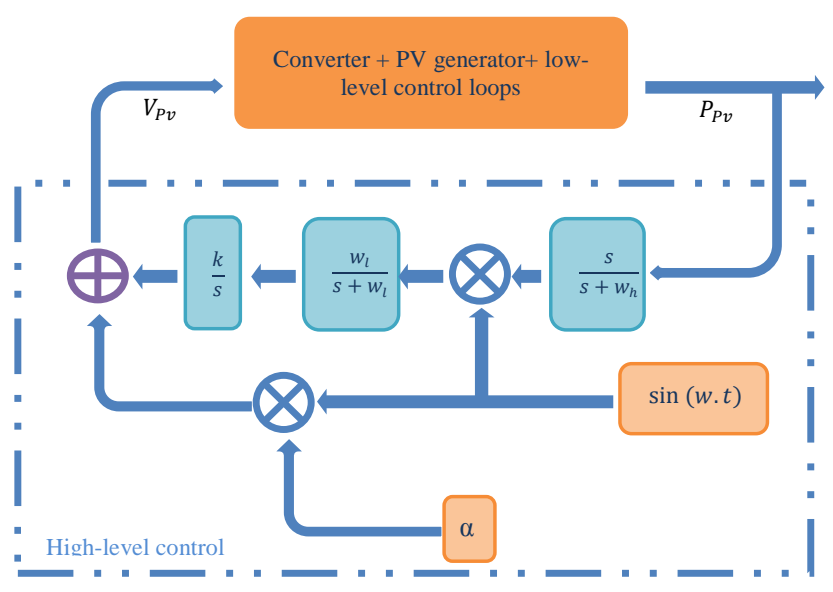

Fig. 6. General ESC algorithm scheme with $\alpha$ being the amplitude of the injected perturbation signal.

\section{B. High-level Control Loop. ESC Design}

Extremum Seeking Control (ESC) is an optimal control strategy aiming at finding the extremum of a unimodal characteristic which varies with time based only on the information that this extremum exists. Needing minimal 
information, ESC is applied when the system is difficult to be modeled, or it is varying significantly for example, because of the surrounding conditions, like the irradiance and the temperature in our case [12].

Although it is proved that this algorithm converges to the maximum power point and can do MPPT successfully when the P-V curve is unimodal [13], but in the case of multiple maxima there is no guarantee that the ESC algorithm will finally succeed in reaching the global maximum. The amplitude of the perturbation signal $\alpha$ plays a main role in this algorithm as it affects both the convergence speed and the variation of the output signal. It is proved that ESC always converges to the MPP even with small amplitude values in the case of unimodal steady-state curves, while in multiple maxima case the ability to overpass local maxima depends on the amplitude value: larger amplitude renders the convergence more probable, but with more ripple in the output signal (trade-off). Different amplitude modulation methods have been proposed to render the algorithm successful also in the case of multiple maxima, namely by forcing escape from local maxima [14].

The general implementation of the ESC algorithm for PV systems is illustrated in Fig. 6.

In order to avoid apply fixed amplitude and having high steady state ripple all the time, two different methods of amplitude modulation are proposed as illustrated in Fig. 7 a and $\mathrm{b}$ respectively.
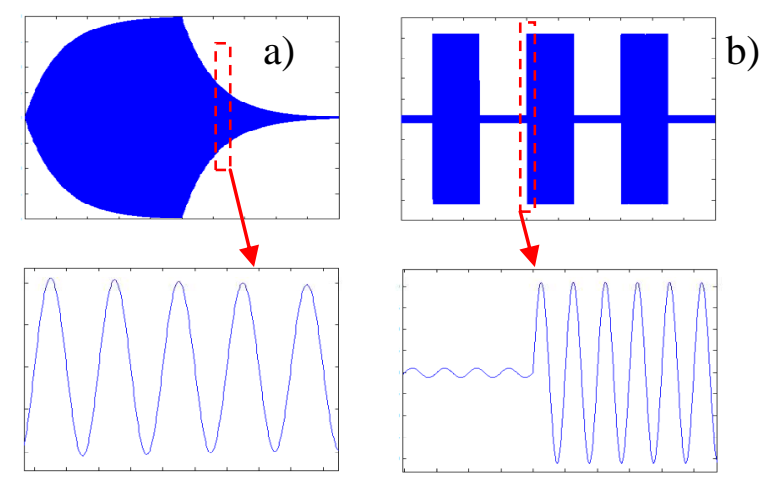

Fig. 7. a) Perturbation signal's amplitude modulation like a first-order system response; b) Perturbation signal's amplitude modulation as $20 \%$ duty-ratio pulses.

The first scheme is to change the amplitude of the perturbation signal, $\alpha$, as the response of a first-order system to a square wave signal (like charging and discharging a capacitor). This amplitude modulation is proved to converge to the global MPP in case of multiple maxima, provided that good parameters tuning has been performed [15].

The second scheme (see Fig. 7b) is an improved version of the previous scheme, where $\alpha$ is changed like pulses with small duty ratio (20\%). The small value is the dominant one since it is applied most of the time, this value guarantees the MPPT of the PV generator power with reasonable ripple.
The larger value is applied periodically in order to ensure that the system never blocks in local maximum.

Note that changing $\alpha$ needs some parameters to be tuned, depending on the system configuration. The worst case scenario should be considered in order to ensure that the system is able to work at the global maximum regardless of the climatic conditions changes (solar irradiance and temperature).

\section{V.SIMULATION RESULTS}

Both ESC algorithms with variable amplitude of perturbation signal $\alpha$ are tested in different sunny and cloudy weather scenarios. The features of the considered PV system are given in Appendix A. In sunny weather, the system has unimodal $P$ - $V$ curve as shown in Fig. 8, thus ESC succeeds in MPPT regardless of the value of $\alpha$, but larger value leads to higher variations of the output power extracted from the PV generator.
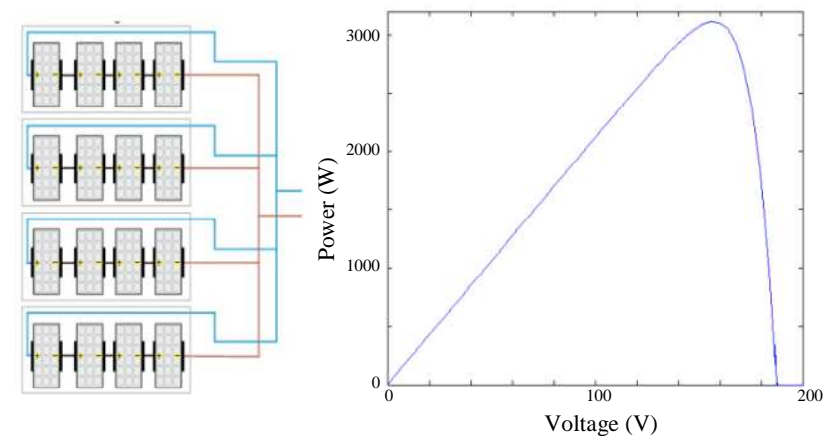

Fig. 8. $P-V$ curve in reference conditions.

In cloudy weather, different scenarios are tested to analyze the tracking performance of the ESC and its ability to find the global MPP. The first scenario is when a cloud passes over the system applying an incremental shadow. The degradation of the total generated power is clearly noticed while the cloud is passing over the PV generator covering more rows of panels. Next, in order to denote the different shadowing status of the PV generator (as consequence of clouds passing over) symbols like the ones represented in Fig. 9 are used.

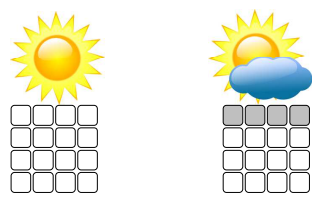

Fig. 9. Symbols describing the shadowing status of the PV generator: a) PV generator is not shadowed; b) first row of the PV generator is shadowed.

The cloud scenario (the irradiance evolutions) is directly reflected in the power time evolution in Fig. 10; the same figure shows comparatively the performance of the two ESC algorithms with amplitude modulation doing MPPT. In Fig. 10 the successive shadowing states due to the cloud passing over have been represented by numbers, whose meaning is explained in Fig. 11. Although this scenario is simulated for just 20 seconds, it could be expanded to reflect the impact of 

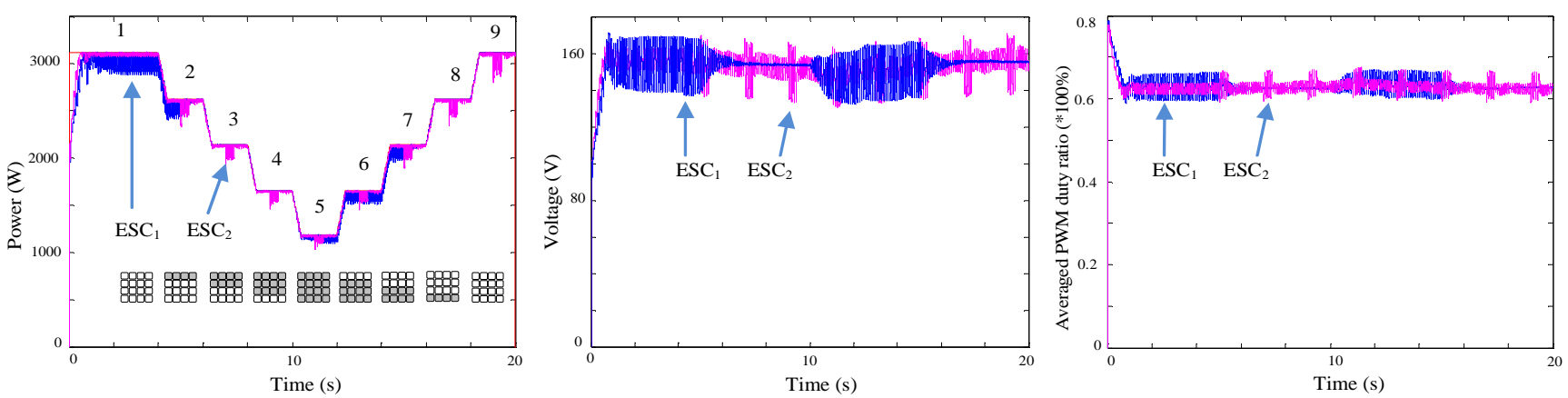

Fig. 10. Cloud passing over the PV generator and its impact on the ESC MPPT performance and evolutions of interest variables (PV power, PV voltage and boost duty ratio): blue: ESC1 with perturbation signal's amplitude, $\alpha$, varying like first-order system response; pink: ESC2 with $\alpha$ varying like pulses.

the cloud on the behavior extended along a whole day.

Both ESC algorithms converge to the MPP in less than 1 second (the convergence speed depends on the amplitude $\alpha$ ). They succeed in tracking the MPP and to work around it all the time. Also, the power ripple generated by both algorithms can be compared, pulses strategy leads to less chattering in output power since $\alpha$ is small most of the time.

The second scenario corresponds to a cloud covering some panels of the PV system. It is supposed that the cloud comes suddenly over the PV generator; such event produces significantly imbalanced solar irradiation, leading to multiple maxima.

Fig. 12 shows the $P-V$ characteristics and how it is affected by the passing of a cloud over the panels.

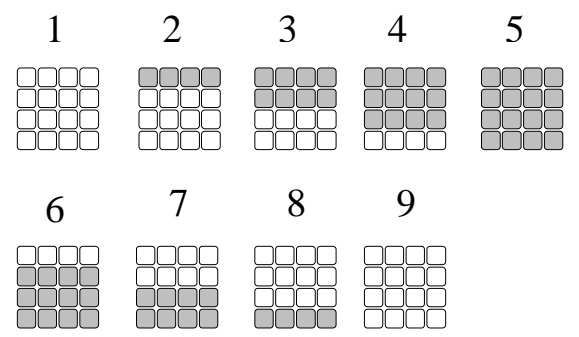

Fig. 11. Sequence of shadowing states of the PV generator corresponding to the scenario used in Fig. 10.

According to the simulation results (Fig.13), both algorithms fail to reach the global maximum since $\alpha$ is not large enough to pass the local maximum. Sufficient increasing of $\alpha$ prevents the system from blocking in local maximum and ensures successful MPPT performance. Fig. 14 shows that the system is working at the global MPP. On the other hand, the second strategy has better results in terms of ripple amplitude where the perturbation signal has high amplitude for a short period of time.

The DC-link power ripple has to be minimized and it appears as a drawback, but one can note that the ripple frequency is precisely known in both cases and depends on the chosen modulation signal and the carrier signal (the perturbation signal frequency is $10 \mathrm{~Hz}$ ). This signal can therefore be eliminated by some filtering action embedded into the DC-AC inverter control. Effective implementation of such action is beyond the scope of this paper

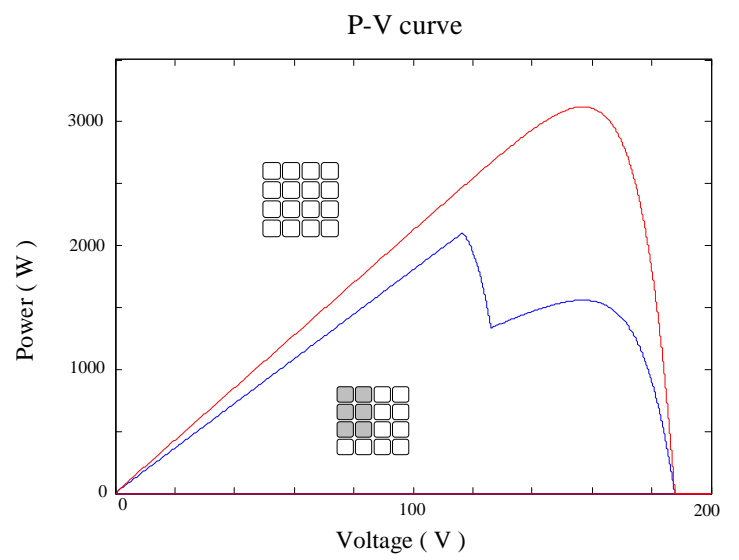

Fig. 12. Significantly imbalanced irradiance distribution leads to multiple maxima in the $P$ - $V$ curve in contrast to the balanced one.

\section{CONCLUSION AND FUTURE WORK}

A modified $\mathrm{P} \& \mathrm{O}$ algorithm is presented in this paper and it has dealt with single-phased grid-connected photovoltaic systems. The main control objective is to harvest the maximum power available irrespective of the solar irradiance and temperature conditions. The performance of the new MPPT algorithm is studied in conditions of strongly imbalanced irradiance levels applied to the PV generator. In such cases multiple maxima may be present in the steadystate power curve and the system must be kept operating close to the global maximum power point. As the amplitude of the searching sinusoidal signal plays the main role in over-passing the local maxima towards the global one, the basic idea is to vary the amplitude of perturbation signal in order to force escaping the local maxima and minimizing the ripple in steady state.

Modulation of this amplitude by two strategies has been analyzed: changing as the response of a first-order system (like charging and discharging a capacitor) and changing as sequence of pulses with small duty ratio. The second strategy has better results due to the short period of high amplitude of the perturbation signal. Most of the simulation 

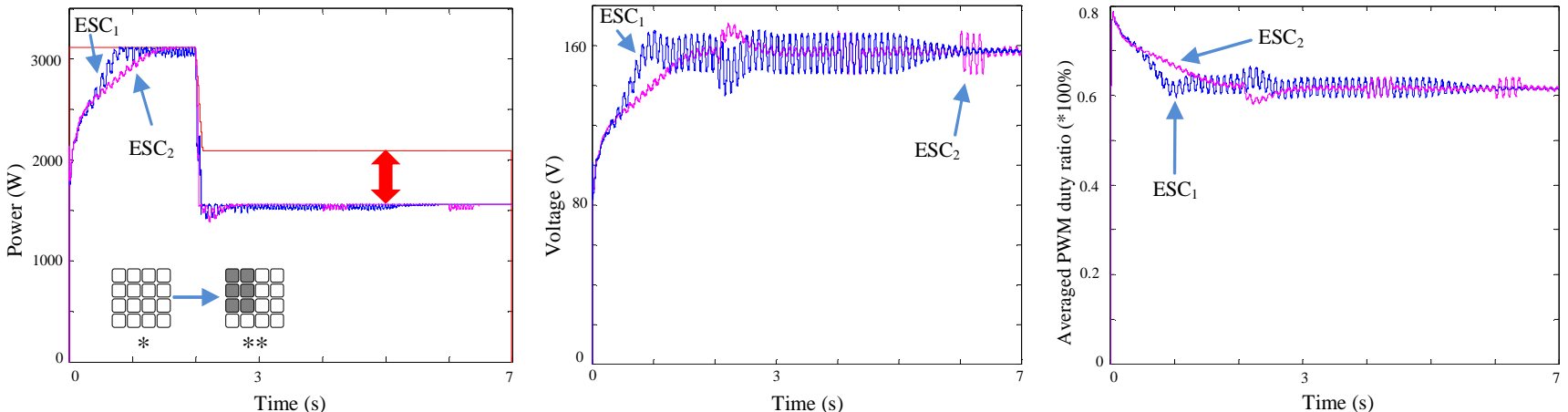

Fig. 13. Evolutions of power, voltage and duty ratio for significantly imbalanced irradiance distribution (step insulation from $* \rightarrow * *$ ) and the unsatisfactory ESC MPPT performance because of small $\alpha$ : blue: ESC1 with $\alpha$ varying like first-order system response; pink: ESC2 with $\alpha$ varying like pulses.
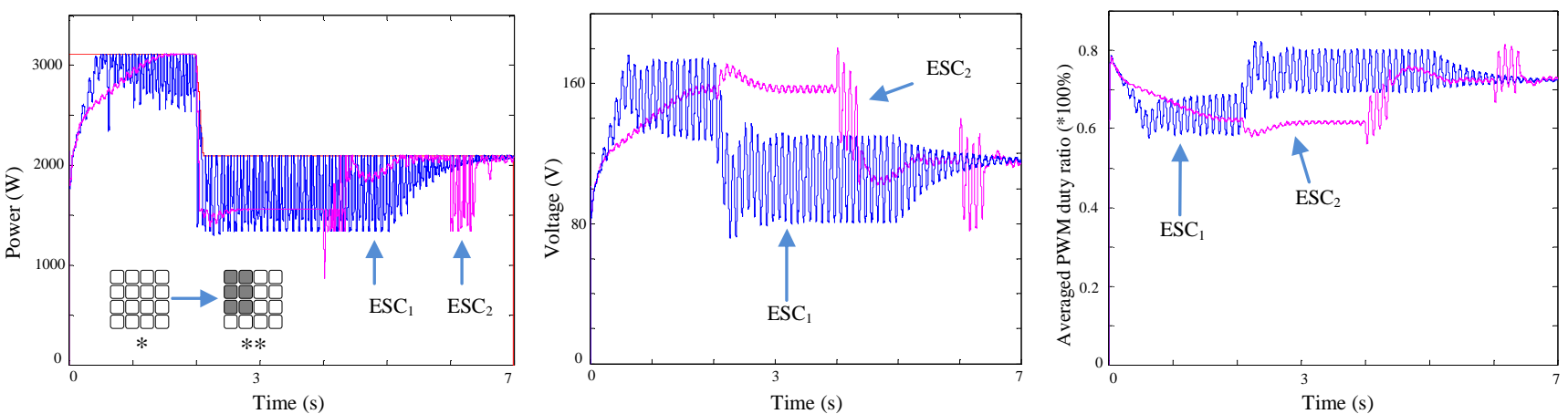

Fig. 14. Evolutions of power, voltage and duty ratio for significantly imbalanced irradiance distribution (step insulation from $* \rightarrow * *$ ) and the MPPT performance after increasing $\alpha$ value: blue: ESC1 with $\alpha$ varying like first-order system response; pink: ESC2 with $\alpha$ varying like pulses.

scenarios show satisfactory results to be applied in real world with good performance. Applying the MPPT-by-ESC strategy to other grid-connected renewable energy sources is an interesting issue to investigate further.

\section{APPENDIX}

$P V$ generator: $3 \mathrm{~kW}$ maximum power in reference conditions (irradiance $1000 \mathrm{~W} / \mathrm{m}^{2}$ and temperature $25^{\circ} \mathrm{C}$ ); total number of cells in one PV panel is 72 and the total number of PV panels is 16 (4*4 matrix); Chopper type: boost, $L_{c h}=0.5 \mathrm{mH}, C_{P V}=270 \mu \mathrm{F}$, switching frequency $10 \mathrm{kHz} ; D C$-link: $V_{D C}=500 \mathrm{~V}, C_{D C}=470 \mu \mathrm{F}$; ESC parameters: probing signal: $10 \mathrm{~Hz}$; integrator parameter $k=2$; wash-out filters: $\omega_{l}=\omega_{h}=2 \pi \mathrm{rad} / \mathrm{s}$

\section{REFERENCES}

[1] K. Kachhiya, M. Lokhande, and M. Patel, "MATLAB/Simulink Model of Solar PV Module and MPPT Algorithm," National Conference on Recent Trends in Engineering \& Technology, May 1314 2011, Gujarat, India.

[2] W. De Soto, "Improvement and Validation of a Model for Photovoltaic Array Performance," M.Sc. Dissertation, University of Wisconsin-Madison, Solar Energy Laboratory, 2004.

[3] M. Wolsink, "The research agenda on social acceptance of distributed generation in smart grids: Renewable as common pool resources," Renewable and Sustainable Energy Reviews, vol. 16, pp. 822-835, 2012.

[4] S. Rustemli, and F. Dincer, "Modeling of Photovoltaic Panel and Examining Effects of Temperature in Matlab/Simulink," Electronics and Electrical Engineering, vol. 109, no. 3, pp. 35-40, 2011.

[5] V. Salas, E. Olías, A. Barrado, and A. Lázaro, "Review of the maximum power point tracking algorithms for stand-alone photovoltaic systems," Solar Energy Materials and Solar Cells, vol. 90, pp. 1555-1578, 2006.

[6] J. Lee, H. Cho, K. Kim and P. Krein, "Irradiance and Temperature Transient Sensitivity Analysis for Photovoltaic Control," 8th
International Conference on Power Electronics - ECCE KOREA, pp. 393-400, 2011.

[7] A. Ghaffari, S. Seshagiri and M. Krstič "Power Optimization for Photovoltaic Micro-Converters using Multivariable Gradient-Based Extremum-Seeking," American Control Conference, Canada, 2012.

[8] H. Malek, S. Dadras, Y. Chen "A Fractional Order Maximum Power Point Tracker: Stability Analysis and Experiments," The 51st IEEE Conference on Decision and Control, USA, 2012.

[9] S. Brunton, C. Rowley, S. Kulkarni and C. Clarkson "Maximum Power Point Tracking for Photovoltaic Optimization Using RippleBased Extremum Seeking Control," IEEE Transactions on Power Electronics, vol. 25, no. 10, October 2010.

[10] A. I. Bratcu, I. Munteanu, S. Bacha, and B. Raison, "Maximum Power Point Tracking of Grid-connected Photovoltaic Arrays by Using Extremum Seeking Control," Control Engineering and Applied Informatics, vol. 10, no. 4, pp. 3-12, 2008.

[11] D. Ocnaşu, "Modeling, Control and Real-time Simulation of Nonconventional Energy Generation Systems," Ph.D. Thesis, Grenoble Institute of Technology, 2008.

[12] K. B. Ariyur, and M. Krstič, Real-Time Optimization by ExtremumSeeking Control. Wiley-Interscience, 2003.

[13] M. Krstič, and H.-H. Wang, "Stability of extremum seeking feedback for general nonlinear dynamic systems," Automatica, vol. 36, pp. 595$601,2000$.

[14] Y. Tan, D. Nešić, I.M.Y. Mareels, and A. Astolfi "Global Extremum seeking in the presence of local extrema" Automatica, vol. 45, no. 1, pp. 245-251, 2009.

[15] A. Kouchaki, H. Iman-Eini, B. Asaei "A new maximum power point tracking strategy for PV arrays under uniform and non-uniform insolation conditions," Solar Energy, vol. 91, pp. 221-232, 2013.

[16] M. Krstič, "Performance improvement and limitations in extremum seeking control," Systems and Control Letters, vol. 39, pp. 313-326, 2000. 